INPLASY

PROTOCOL

To cite: Cechinel et al.

Association of delirium and fragility in hospitalized elderly: systematic review. Inplasy protocol 202190022. doi: 10.37766/inplasy2021.9.0022

Received: 07 September 2021

Published: 07 September 2021

Corresponding author:

Clovis Cechinel

cechinelc@hotmail.com

Author Affiliation:

Universidade Federeal do

Paraná

Support: None.

Review Stage at time of this submission: The review has not yet started.

Conflicts of interest:

None declared.

\section{ASSOCIATION OF DELIRIUM AND FRAGILITY IN HOSPITALIZED ELDERLY: SYSTEMATIC REVIEW}

Cechinel, C1; Rodrigues, JAM².

Review question / Objective: What is the relationship between delirium and frailty in hospitalized elderly people? The objective of this research is to analyze the association between frailty and delirium in hospitalized elderly people, through a systematic literature review.

Condition being studied: Frailty and delirium in hospitalized aged.

Information sources: A specific search strategy for the language of each database was developed using, initially, the Medical Subject Headings (MEsH) descriptor and later translated to specific descriptors (Descriptors in Health Sciences (DeCS) and Embase Subject Headings (Emtree)). The search strategy will be applied by the researchers in the MEDLINE databases through the Pubmed Portal; Scielo; VHL; EMBASE, CINAHL, Scopus and Web of Science through the CAPES Journal Portal; CENTRAL via Cochrane.

INPLASY registration number: This protocol was registered with the International Platform of Registered Systematic Review and Meta-Analysis Protocols (INPLASY) on 07 September 2021 and was last updated on 07 September 2021 (registration number INPLASY202190022).

\section{INTRODUCTION}

Review question / Objective: What is the relationship between delirium and frailty in hospitalized elderly people? The objective of this research is to analyze the association between frailty and delirium in hospitalized elderly people, through a systematic literature review.

Condition being studied: Frailty and delirium in hospitalized aged. 


\section{METHODS}

Participant or population: People over 60 years old.

\section{Intervention: None.}

Comparator: delirium and without delirium.

Study designs to be included: Observational studies.

Eligibility criteria: presence of frailty and delirium, hospitalized, age $\geq 60$ years.

Information sources: A specific search strategy for the language of each database was developed using, initially, the Medical Subject Headings (MEsH) descriptor and later translated to specific descriptors (Descriptors in Health Sciences (DeCS) and Embase Subject Headings (Emtree)). The search strategy will be applied by the researchers in the MEDLINE databases through the Pubmed Portal; Scielo; VHL; EMBASE, CINAHL, Scopus and Web of Science through the CAPES Journal Portal; CENTRAL via Cochrane.

Main outcome(s): To verify the prevalence go frailty in hospitalized elderly and what os the relationship of frailty to delirium development. Once the data record is released, it will search the databases and, if possible, perform a meta-analysis.

Quality assessment / Risk of bias analysis: Newcastle-Ottawa scale.

Strategy of data synthesis: Information including age, percentage of female, authors, years, country, participant type, frailty instruments, delirium criteria, sample size and data. Data will independent extracted by 2 authors.

Subgroup analysis: We will conduct a subgroup based in delirium instruments to show if frail hospitalized patients are in increased risk for delirium.

Sensitivity analysis: To assess if the study results were stable, we will conduct a sensitivity analyses and will find if the pooled effects size did not have a material change.

Country(ies) involved: Brazil.

Keywords: delirium, Frailty, Aged, Hospitalized patient, inpatient.

Contributions of each author:

Author 1 - Clovis Cechinel - Database research, duplicates removing, article titles and abstracts evaluation and ineligible articles remotion, writing of an article and analyses.

Email: cechinelc@hotmail.com

Author 2 - Joao Alberto Martins Rodrigues - Database research, duplicates removing, article titles and abstracts evaluation and ineligible articles remotion, and writing of an article and analyses. 